\title{
Analysis of Separate Isolates of Bordetella pertussis Repeated DNA Sequences
}

\author{
By WILLIAM L. MCPHEAT, ${ }^{*}+$ JENNIFER H. HANSON,${ }^{1}$ IAN LIVEY ${ }^{3} \ddagger$ \\ AND JAMES S. ROBERTSON ${ }^{2}$ \\ Divisions of Bacteriology $y^{1}$ and Virology ${ }^{2}$, National Institute for Biological Standards and Control, \\ South Mimms, Hertfordshire EN6 3QG, UK \\ Molecular Genetics Laboratory ${ }^{3}$, PHLS, Centre for Applied Microbiology and Research, \\ Porton Down, Salisbury, Wiltshire SP4 OJG, UK
}

(Received 7 February 1989; accepted 8 March 1989)

Two independent isolates of a Bordetella pertussis repeated DNA unit were sequenced and shown to be an insertion sequence element with five nucleotide differences between the two copies. The sequences were $1053 \mathrm{bp}$ in length with near-perfect terminal inverted repeats of $28 \mathrm{bp}$, had three open reading frames, and were each flanked by short direct repeats. The two insertion sequences showed considerable homology to two other $B$. pertussis repeated DNA sequences reported recently: IS 481 and a $530 \mathrm{bp}$ repeated DNA unit. The $B$. pertussis insertion sequence would appear to comprise a group of closely related sequences differing mainly in flanking direct repeats and the terminal inverted repeats. The two isolates reported here, which were from the adenylate cyclase and agglutinogen 2 regions of the genome, were numbered IS481v1 and IS481v2 respectively.

\section{INTRODUCTION}

Prokaryotic insertion sequence (IS) elements are one class of mobile genetic elements and represent one of several types of repeated DNA sequences (Iida et al., 1983). IS elements are generally $700-1800 \mathrm{bp}$ in size with perfect or near perfect terminal inverted repeats of 10-40 bp, have two to three open reading frames (ORFs; encoding transposition functions only) arranged in a characteristic manner, and are flanked by short direct repeats $(2-12 \mathrm{bp})$ formed on insertion of the IS element at its target site (Calos \& Miller, 1980; lida et al., 1983). The length, but not the sequence, of these direct repeats is thought to be characteristic for each IS element. IS elements frequently affect gene expression by mediating a variety of DNA rearrangements (Iida et al., 1983).

Bordetella pertussis, a respiratory pathogen responsible for whooping cough, has been shown to carry a repeated DNA sequence which was suggested to be an IS element and which was absent from other Bordetella species (McPheat \& McNally, 1987a,b). To characterize fully this repeat unit, two separate copies were sequenced and we confirm here that this repeat unit is an IS element.

During the preparation of this manuscript, two reports were published on the nucleotide sequence analysis of repeated DNA units specific to B. pertussis. McLafferty et al. (1988) reported the isolation of two distinct repeat units, one of which was shown to be a $1053 \mathrm{bp}$ IS element (IS481), whilst Park et al. (1988) reported the presence of a 530 bp repeat unit which had no apparent structural features characteristic of IS elements. The relationships between the reported repeat units and the IS elements sequenced in this report are discussed.

† Present address: ICI Diagnostics, Gadbrook Park, Rudheath, Northwich, Cheshire CW9 7RA, U.K.

$\ddagger$ Present address: Immuno AG, Research Centre, A-2304 Orth/Donau, Austria.

Abbreviations: IS, insertion sequence; ORF, open reading frame.

0001-5400 (C) 1989 SGM 


\section{METHODS}

Subcloning and extraction for DNA sequence determination. The isolation of the two clones, pIL60 and pIL22, each of which carry a separate copy of the repeat DNA sequence, has been detailed in full previously (Livey et al., 1987; McPheat \& McNally, 1987a). A $2.6 \mathrm{kbp} \mathrm{BamHI}$ fragment of pIL60 which carried the repeat unit (McPheat $\& \mathrm{McNally}, 1987 \mathrm{a}$ ) was digested with Clal to provide two fragments of 1.5 and $1 \cdot 1 \mathrm{kbp}$. These were isolated from an agarose gel and were subcloned after digestion by NarI, Sau3AI or TaqI (Northumbria Biologicals) followed by ligation into the pSP6. 4 cloning vector (Melton et al., 1984). The sequence determination of a $2.6 \mathrm{kbp} \mathrm{ClaI-SacI}$ fragment of pIL22 was described by Livey et al. (1987). A contiguous SphI-ClaI fragment of 780 bp from pIL22 was isolated from an agarose gel, digested with NarI, Sau3AI or TaqI and subcloned into pSP6.4. Recombinant plasmids were transformed into Escherichia coli DH5 (Gibco-BRL, Frozen Competent Cells) and transformants selected on Nutrient Agar (Oxoid) containing ampicillin (Sigma) at $50 \mu \mathrm{g} \mathrm{ml}^{-1}$. Single transformed colonies were inoculated into $10 \mathrm{ml} \mathrm{LB}$ medium with ampicillin (Maniatis et al., 1982) and incubated at $37^{\circ} \mathrm{C}$ overnight. Plasmid DNA was prepared either by the methods of Ish-Horowicz \& Burke (1981) or Holmes \& Quigley (1981), treated with RNAase A (Sigma), extracted once with phenol and passed over Sephadex G50 by the spun column procedure (Maniatis et al., 1982), or by $\mathrm{CsCl}$ gradient centrifugation.

DNA sequencing and analysis. Sequencing reactions were performed by the dideoxy chain termination procedure (Sanger et al., 1977) as modified for plasmid DNA by Korneluk et al. (1985) using either Klenow enzyme or a Sequenase kit (United States Biochemical Corporation). Each insert was sequenced on both strands using either the M13 reverse sequencing primer (Pharmacia) or the SP6 sequencing primer (Gibco-BRL). To confirm the junctions of fragments at the ClaI sites of pIL60 and pIL22, synthetic oligonucleotides which fiank the ClaI sites were used as primers. Each nucleotide in the final sequence had been determined at least twice on both strands. The Staden-Plus programs (Staden, 1986) on a VAX computer system, were used to overlap, assemble and analyse the final DNA sequences.

\section{RESULTS AND DISCUSSION}

\section{Structural and sequence analysis of the repeat unit}

To determine the nucleotide sequence of the repeat unit, two separate genomic fragments having in common a single copy each of the repeat unit were sequenced in full. Our first isolate of the repeat unit (RS1) was on pIL60, a $19.6 \mathrm{kbp}$ EcoRI fragment from the adenylate cyclase region cloned into pBR322 (McPheat \& McNally, 1987a). RS1 was localized to a $2.6 \mathrm{kbp}$ Bam HI fragment of pIL60 which was isolated and subcloned for nucleotide sequencing (Fig. 1).

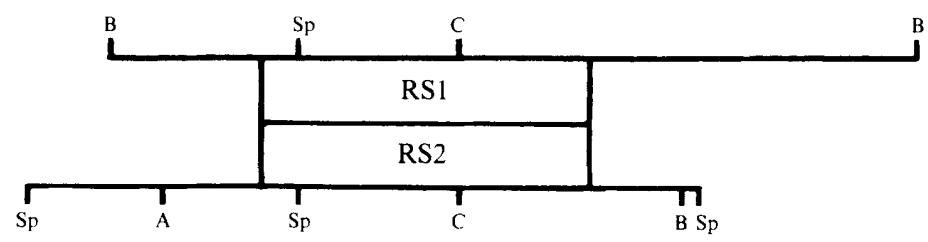

\section{$200 \mathrm{bp}$}

Fig. 1. Fragments sequenced from pIL60 and pIL22 aligned to show location of RS1 and RS2. The upper line is the $2.6 \mathrm{kbp} \mathrm{BamHI}$ fragment from pIL60 (McPheat \& McNally, 1987 a) carrying RS1; the lower line is a contiguous $S p h I$ region of $2.2 \mathrm{kbp}$ from pIL22 (Livey et al., 1987) carrying RS2. The fragments have been aligned to show the homologous regions representing RS1 and RS2 (open boxes). Restriction enzyme sites shown are: A, ApaI; B, BamHI; C, ClaI; Sp, SphI.

Fig. 2. Nucleotide sequence of RS1 and comparison with those of RS2, IS481 and a 530 bp repeated DNA unit from B. pertussis. The nucleotide sequence of RS1 is shown in full on the top line. Nucleotide differences in RS2, IS481 (McLafferty et al., 1988), and the 530 bp repeated unit of Park et al. (1988) (indicated as PARK on the left-hand side) are shown below the sequence of RS1. Nucleotide 777 of RS1 was absent from the PARK sequence as shown by the dot $(O)$. The 530 bp unit started at position 524 of RS1 and the limits of the sequence are shown (()). The $28 \mathrm{bp}$ terminal inverted repeats are indicated by arrows; the ClaI and $S p h I$ sites are overlined. The start and stop codons of the three ORFs are shown by boxes. Flanking genomic sequences are shown in lower case letters with the flanking direct repeats of RS1 and RS2 indicated by arrows. 


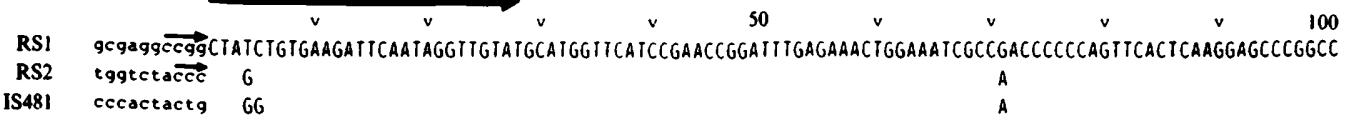

$\begin{array}{rlllllll}\text { ORF } 1 & S p h l & v & v & v & v & v & v\end{array}$

RS

RS2

IS481

RSI

RS2

IS48I

RSI

RS2

IS481

RSI

RS2

IS48 I

RSI

RS2

IS48I

PARK

RSI

RS2

IS48

PARK

RS।

RS2

IS48

PARK

RSI

RS2

IS48]

PARK

RSI

RS2

IS48 I

PARK

RS

RS2

IS48 I

PARK

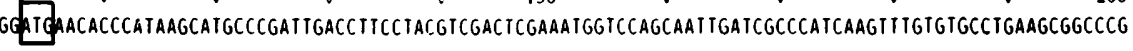

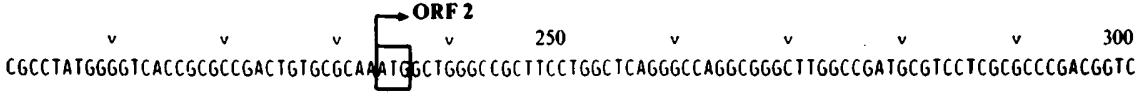

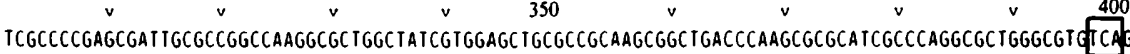

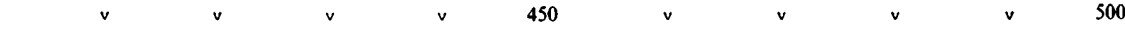
CCAGCACCGTCAGCCGCGTCCTGGCCCGCGCCGGICTGTCGCACCTGGCCGACCTGGAGCCGGCCGAGCCGGTGGTGCGCTACGAGCATCAGGCCCCCGG G



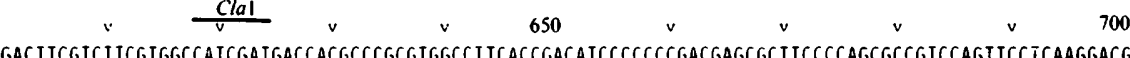

A
A

CAGTGGCCTACTACCAGCGCCIGGGCGTGACCATCCAGCGCTIGCICACCGACAATGGCTCGGCCTITICGCAGCCGCGCCTICGCCGCGCTGTGOCAT

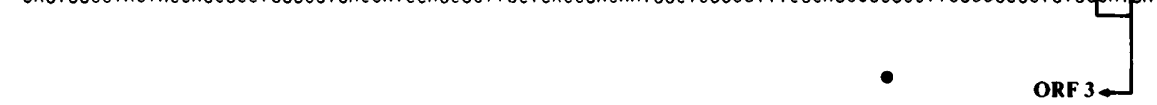

$\begin{array}{lllllllll}\text { v } & v & v & \text { v } & 850 & \text { v } & \text { v } & \text { v } & \text { v }\end{array}$ GCIGGGCATCAAGCACCGCIITACCCGACCITACCGCCCACAGACCAAIGGCAAGGCCGAACGCITCATCCAGTCGGCCTIGCGIGAGTGGGCTIACGCT

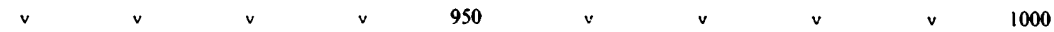
CACACCTACCAGAACICCCAACACCGAGCCGAIGCCATGAAATCCIGGCIACACCACTACAACIGGCAICGACCCCACCAAGGCATCGGGCGCGCTGTAC

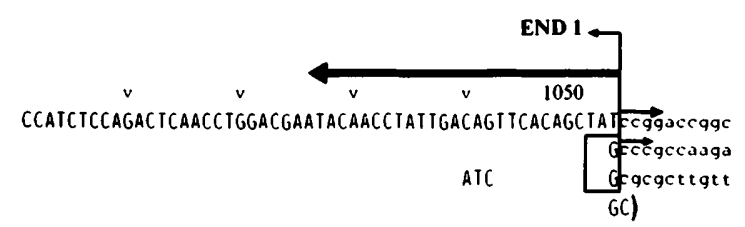


The second copy of the repeat unit (RS2) was on pIL22, a $7.5 \mathrm{kbp}$ chromosomal fragment carrying the structural subunit gene for the serotype 2 fimbrial agglutinogen (agglutinogen 2) of B. pertussis cloned into pBR328 (Livey et al., 1987). Hybridization of the BamHI subclone of RS1 to restriction digests of pIL22 showed that the two clones had only the repeated DNA sequence in common (data not shown). To determine the nucleotide sequence of RS2, a $2 \cdot 2 \mathrm{kbp}$ region of pIL22 consisting of two adjacent SphI fragments was sequenced (Fig. 1).

Comparison of the sequences of the $2.6 \mathrm{kbp}$ and $2.2 \mathrm{kbp}$ fragments identified a homologous region of 1053 bp representing the repeated DNA units RS1 and RS2 (Fig. 1, Fig. 2). The G + C base content of the repeat unit was $63.9 \%$ which was similar to that reported for the genes encoding pertussis toxin, agglutinogen 2, and adenylate cyclase (Locht \& Keith, 1986; Livey et al., 1987; Glaser et al., 1988). The structural features of this 1053 bp sequence, its length, nearperfect terminal inverted repeats of $28 \mathrm{bp}$, three ORFs in a typical arrangement, and flanking short direct repeats of 3 or 4 bp (Fig. 2), were all consistent with those of IS elements.

Both RS1 and RS2 had near-perfect terminal inverted repeats of 28 bp which could hybridize to form the stem-loop structures shown in Fig. 3. Within the terminal inverted repeats of RS1 and RS2 there was an identical $3 \mathrm{bp}$ mismatch. RS1 had two further mismatches resulting from $\mathrm{G} \rightarrow \mathrm{T}$ transversions. The nucleotide sequences immediately flanking the termini of RS1 and RS2 are also shown in Fig. 3. A 4 bp direct repeat (CCGG) flanks the copy of RS1, whilst a 3 bp direct repeat (CCC) flanks the copy of RS2. Flanking direct repeats of this size are shared by other IS elements such as IS3 ( 3 or 4 bp), IS3411 (3 bp), IS5 (4 bp), IS51 (3 bp), and IS52 (4 bp) (Iida et al., 1983; Yamada et al., 1986; Ishiguro \& Sato, 1988).

Computer analysis of the sequences of RS1 and RS2 identified three main ORFs with the potential to code for proteins of 316,117 and 133 amino acids respectively (Fig. 2). ORF1 in RS1 had no stop codon at the end of the repeat unit and extended a further 1057 nucleotides (352 amino acids) beyond the end of the restriction fragment with no intervening stop codon. ORF3
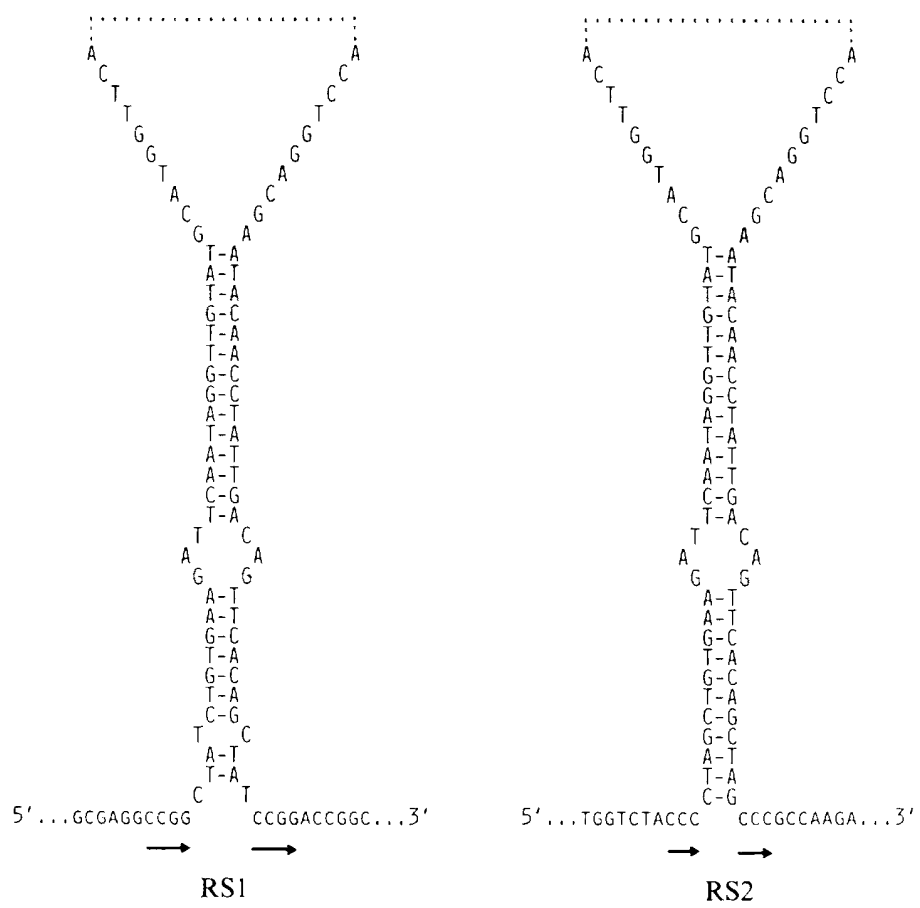

Fig. 3. Terminal inverted repeats and flanking sequences of RS1 and RS2. The stem-loop structure formed by maximum hybridization is shown. Flanking genomic sequences are shown in smaller letters. The 3 and 4 bp direct repeats flanking RS2 and RS1 respectively are underlined with arrows. 
was internal to ORF1, on the opposite strand and in the same frame configuration (codons in phase). This arrangement of ORFs is typical of IS elements (Iida et al., 1983). Although it is not known which, if any, of these ORFs are transcribed in vivo, a putative promoter (nucleotides 5-31), transcriptional start site (nucleotides 37-39), and ribosome binding site (nucleotides 89-94) were identified preceding ORF1 (not shown in Fig. 2). A computer search of the appropriate EMBL databases for homologies to the amino acid sequences of these three ORFs revealed no significant homology.

\section{Comparison with other $B$. pertussis repeated DNA sequences}

The nucleotide sequences of two further $B$. pertussis repeated DNA units have been published recently (McLafferty et al., 1988; Park et al., 1988) and are shown in comparison to those of RS1 and RS2 in Fig. 2. McLafferty et al. (1988) presented the sequence of a $1053 \mathrm{bp}$ IS element (IS481) which they suggested may be the same as the repeated DNA unit isolated by McPheat \& McNally (1987a). The sequence comparison in Fig. 2 shows that RS1, RS 2 and IS481 are very closely related IS elements. RS1 differed from RS2 at five positions (two within the terminal inverted repeats) and from IS481 at eight positions (six within the terminal inverted repeats); RS2 and IS481 differed at five positions, four of which were within the terminal inverted repeats. All three sequences encoded the same three ORFs, although ORF1 was not mentioned by McLafferty et al. (1988). IS481 differed from RS1 and RS2 in having no flanking direct repeat sequences (Fig. 2). The absence of short direct repeat sequences flanking IS elements has been found in a few other instances and may have resulted from mutations arising subsequent to insertion of the element (Iida et al., 1983; Barberis-Maino et al., 1987).

Park et al. (1988) reported the nucleotide sequence of a 530 bp repeat unit from B. pertussis. This repeat unit had no obvious structural features, but analysis of the DNA sequence showed that it matched almost exactly with the right terminal $530 \mathrm{bp}$ of RS1, RS2 and IS481 (nucleotides 524-1053; Fig. 2). Two of the three clones from which the 530 bp sequence was obtained showed further, variable, homology to RS1, RS2 and IS481 in the preceding 270 nucleotides (not shown in Fig. 2). It is clear that the 530 bp sequence described by Park et al. (1988) is closely related to RS1, RS2 and IS481, but it remains to be shown whether it is a naturally occurring deletion product of the $1053 \mathrm{bp}$ IS element or if there is some alternative explanation.

Analysis of the sequences adjacent to the four repeated DNA units compared here shows that each was from a different chromosomal site; also, RS1 and RS2 at least were isolated from separate strains of $B$. pertussis (Tohama and Wellcome 28 respectively). These factors could account for the differences in nucleotides and flanking direct repeats between the sequences. However, the nucleotide differences appeared to be concentrated in the terminal inverted repeats, which are thought to be involved in the transposition of IS elements, and this could have implications for the mobility of individual members of the group (Iida et al., 1983).

Thus, RS1, RS2 and IS481 form a closely related group of IS elements and accordingly RS1 and RS2 have been designated IS481v1 and IS481v2 respectively, as suggested to us by the Plasmid Reference Centre (Lederberg, 1987).

The authors would like to thank Martha McLafferty (University of Virginia) for preview and discussions of her IS481 sequence data prior to publication, Graham Wells and Carolyn Nicolson for nucleotide sequence analysis, and Mike Squire for the synthesis of oligonucleotide sequencing primers.

\section{REFERENCES}

Barberis-Maino, L., Berger-BÄChI, B., Weber, H., BECK, W. D. \& KAYSER, F. H. (1987). IS431, a staphylococcal insertion sequence-like element related to IS26 from Proteus vulgaris. Gene 59, 107113.

Calos, M. P. \& Miller. J. H. (1980). Transposable elements. Cell 20, 579-595.

Glaser, P., Ladant, D., Sezer, O., Pichot, F., Ullmann, A. \& Danchin, A. (1988). The calmodu- lin-sensitive adenylate cyclase of Bordetella pertussis: cloning and expression in Escherichia coli. Molecular Microbiology 2, 19-30.

Holmes, D. S. \& Quigley, M. (1981). A rapid boiling method for the preparation of bacterial plasmids. Analytical Biochemistry 114, 193-197.

IIDA, S., MeYer, J. \& ARBER, W. (1983). Prokaryotic IS elements. In Mobile Genetic Elements, pp. 159-221. Edited by J. A. Shapiro. London: Academic Press. 
IsH-Horowicz, P. \& Burke, J. F. (1981). Rapid and efficient cosmid cloning. Nucleic Acids Research 9, 2989-2998.

ISHiguro, N. \& SATo, G. (1988). Nucleotide sequence of insertion sequence IS3411, which flanks the citrate utilization determinant of Transposon Tn3411. Journal of Bacteriology 170, 1902-1906.

Korneluk, R. G., Quan, F. \& Gravel, R. A. (1985). Rapid and reliable dideoxy sequencing of doublestranded DNA. Gene 40, 317-323.

LeDerberG, E. M. (1987). Plasmid Reference Centre Registry of transposon ( $\mathrm{Tn}$ ) and insertion sequence (IS) allocations through December 1986. Gene 51, 115-118.

Livey, I., Duggleby, C. J. \& Robinson, A. (1987). Cloning and nucleotide sequence analysis of the serotype 2 fimbrial subunit gene of Bordetella pertussis. Molecular Microbiology 1, 203-209.

LOCHT, C. \& KeITH, J. M. (1986). Pertussis toxin gene: nucleotide sequence and genetic organization. Science 232, 1258-1264.

Maniatis, T., Fritsch, E. F. \& SAMBrooK, J. (1982). Molecular Cloning: a Laboratory Manual. Cold Spring Harbor, NY: Cold Spring Harbor Laboratory.

MCLAFferty, M. A., Harcus, D R. \& Hewlett, E. L. (1988). Nucleotide sequence and characterization of a repetitive DNA element from the genome of Bordetella pertussis with characteristics of an insertion sequence. Journal of General Microbiology 134, 2297-2306.
MCPheat, W. L. \& MCNALLY, T. (1987a). Isolation of a repeated DNA sequence from Bordetella pertussis. Journal of General Microbiology 133, 323-330.

McPheat, W. L. \& MCNally, T. (1987b). Phase I and phase IV strains of Bordetella pertussis carry a repeated DNA sequence not found in other Bordetella species. FEMS Microbiology Letters 41, 357360.

Melton, D. A., Krieg, P. A., Rebagliati, M. R., Maniatis, T., ZinN, K. \& Green, M. R. (1984). Efficient in vitro synthesis of biologically active RNA and RNA hybridization probes from plasmids containing a bacteriophage SP6 promoter. Nucleic Acids Research 12, 7035-7056.

Park, I., Saurin, W. \& Ullmann, L. A. (1988). A highly conserved 530 base-pair repeated DNA sequence specific for Bordetella pertussis. FEMS Microbiology Letters 52, 19-24.

Sanger, F., Nicklen, S. \& Coulson, A. R. (1977). DNA sequencing with chain-terminating inhibitors. Proceedings of the National Academy of Sciences of the United States of America 74, 5463-5467.

STADEN, R. (1986). The current status and portability of our sequence handling software. Nucleic Acids Research 14, 217-231.

YAMADA, T., LeE, P.-D. \& Kosuge, T. (1986). Insertion sequence elements of Pseudomonas savastanoi: nucleotide sequence and homology with Agrobacterium tumefaciens transfer DNA. Proceedings of the National Academy of Sciences of the United States of America 83, 8263-8267. 